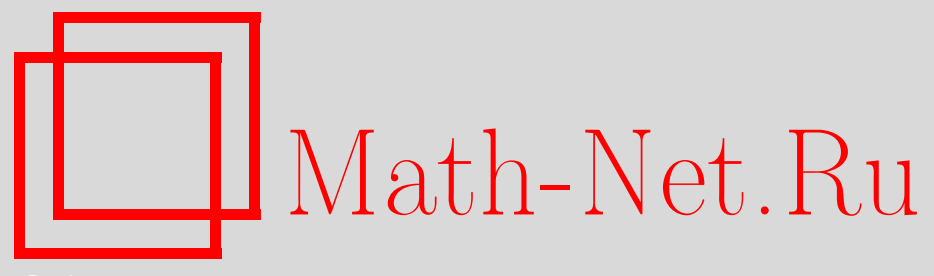

А. С. Ляпин, Усреднение траекторного аттрактора нелинейного волнового уравнения с быстро осциллирующей правой частью, Матем. заметки, 2007, том 82, выпуск $3,390-394$

DOI: https://doi.org/10.4213/mzm3851

Использование Общероссийского математического портала Math-Net.Ru подразумевает, что вы прочитали и согласны с пользовательским соглашением http://www . mathnet.ru/rus/agreement

Параметры загрузки:

IP: 54.209 .52 .79

26 апреля 2023 г., 14:18:04

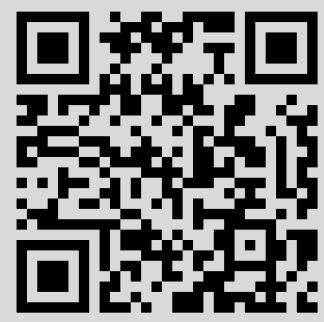


Том 82 выпуск 3 сентябрь 2007

\section{Усреднение траекторного аттрактора нелинейного волнового уравнения с быстро осциллирующей правой частью}

\section{А. С. Ляпин}

Рассматривается нелинейное неавтономное гиперболическое уравнение с диссипацией и с малым параметром при старшей производной по времени. В этом уравнении есть также быстро осциллирующая внешняя сила. Используя стандартную технику построения траекторного аттрактора, доказывается сходимость аттрактора нелинейного неавтономного гиперболического уравнения с диссипацией к аттрактору соответствующего параболического уравнения.

Библиография: 2 названия.

Рассматривается нелинейное волновое уравнение вида

$$
\varepsilon^{2} u_{t t}+\gamma u_{t}+f(t ; u)=\Delta u+g(t / \varepsilon ; x),
$$

где $x \in \Omega \Subset \mathbb{R}^{n}, \gamma>0$ и $0<\varepsilon \leqslant 1$. Предполагается, что $\left.u\right|_{\partial \Omega}=0$. В работе [1] показано, что для любого $\varepsilon>0$ при выполнении некоторых условий относительно функции $f(t ; u)$ уравнение $(1)$ имеет траекторный аттрактор $A^{(\varepsilon)}$. В настоящей работе исследуется поведение $A^{(\varepsilon)}$ при $\varepsilon \rightarrow 0$. Для этого наряду с уравнением (1) изучается предельное параболическое уравнение

$$
\gamma \bar{u}_{t}+f(t ; \bar{u})=\Delta \bar{u}+\bar{g}(x) .
$$

Уравнение (2), как показано в [1], имеет траекторный аттрактор $A$. Мы докажем, что в соответствующей топологии любая окрестность $A$ содержит $A^{(\varepsilon)}$, если $\varepsilon$ достаточно мало.

Перейдем теперь к точным формулировкам. Относительно функции $f(t ; v)$ и ее первообразной $F(t ; u)=\int_{0}^{u} f(t ; v) d v$ предполагается, что

$$
\begin{gathered}
F(t ; v) \geqslant \gamma_{1}|v|^{p}-C_{1}, \\
C_{3}|v|^{p}-C_{4} \leqslant f(t ; v) v \leqslant C_{5}|v|^{p}-C_{6}, \\
\alpha f(t ; v) v-\frac{\partial F(t ; v)}{\partial t} \geqslant \alpha \gamma_{2} F(t ; v)-C_{2} .
\end{gathered}
$$

Здесь $\alpha>0, C_{i}>0, \gamma_{i}>0$. Внешняя сила $g(t / \varepsilon ; x)$ удовлетворяет условию

$$
\int_{t}^{t+1}\|g(\tau / \varepsilon ; x)\|_{L_{2}(\Omega)}^{2} d \tau<C<\infty \quad \forall t \in \mathbb{R}
$$

(C) А. С. Ляпин, 2007 
равномерно по $0<\varepsilon \leqslant 1$. Для уравнения (1) при $t=\tau$ задаются начальные условия

$$
\begin{aligned}
\left.u\right|_{t=\tau} & =u_{0} \in H_{0}^{1}(\Omega) \cap L_{p}(\Omega), \\
\left.u_{t}\right|_{t=\tau} & =u_{1} \in L_{2}(\Omega) .
\end{aligned}
$$

Теорема 1. Пусть выполнены условия (3)-(6). Тогда для любого $M>0$ задача (1),(7) имеет хотя бы одно решение $u(x ; t) \in L_{\infty}\left([0 ; M] ; H_{0}^{1} \cap L_{p}(\Omega)\right), u u_{t} \in$ $L_{\infty}\left([0 ; M] ; L_{2}(\Omega)\right)$. При этом имеет место следующее неравенство:

$$
\begin{gathered}
Z(t) \leqslant C Z(0) e^{-\kappa t}+R_{\kappa} \\
Z(t)=\|u\|_{H_{0}^{1}(\Omega)}^{2}+\|u\|_{L_{p}(\Omega)}^{p}+\left\|u_{t}\right\|_{L_{2}(\Omega \times[t ; t+1])}^{2} .
\end{gathered}
$$

Здесъ $\kappa>0$ и $R_{\kappa} \geqslant 0$; эти числа зависят от $\alpha, C, \gamma$, но не зависят от $\varepsilon$.

Доказательство теоремы проводится как обычно методом Галеркина (см., например, [1]). Для этого рассматривается вспомогательная функция $V=u_{t}+\alpha u$. Умножив уравнение (1) на $V$, после простых преобразований получаем неравенство (8). Отсюда легко выводится существование решения задачи (1), (7).

Ввиду того, что условия (3)-(6) не гарантируют единственности решения задачи $(1),(7)$, для изучения поведения $u(x ; t)$ при $t \rightarrow \infty$ используется траекторный аттрактор, который строится ниже. Для этого нам понадобятся следующие пространства.

ОПРЕДЕЛЕНИЕ 1. Пусть $\Xi=\Xi_{1} \times \Xi_{2}$, где

$$
\begin{aligned}
& \Xi_{1}=\left\{\varphi(t ; u) \in C^{\text {loc }}\left(\mathbb{R} ; C^{\text {loc }}(\mathbb{R})\right) \mid \partial \varphi / \partial t \in C^{\text {loc }}\left(\mathbb{R} ; C^{\text {loc }}(\mathbb{R})\right)\right\}, \\
& \Xi_{2}=L_{2 ; w}^{\text {loc }}\left(\mathbb{R} ; L_{2 ; w}(\Omega)\right) .
\end{aligned}
$$

Следует отметить, что $\sigma_{k}=\left(\varphi_{k}(t ; u) ; \psi_{k}(t ; x)\right)$ сходится к $\sigma=(\varphi(t ; u) ; \psi(t ; x))$ в $\Xi$, если для любых фиксированных чисел $r>0$ и $M>0$

1) $\varphi_{k}(t ; u) \rightarrow \varphi(t ; u)$ и $\partial \varphi_{k} / \partial t \rightarrow \partial \varphi / \partial t$ в пространстве $C([-M ; M] \times[-r ; r])$;

2) $\psi_{k}(t ; x)$ слабо сходится к $\psi(t ; x)$ в $L_{2}(\Omega \times[-M ; M])$.

Сформулируем теперь понятие трансляционно-компактной функции.

ОПРЕДЕЛЕНИЕ 2. Функция $f(t ; u)$ называется трансляционно-компактной в пространстве $\Xi_{1}$, если оболочка $\mathscr{H}(f(t ; u))$, задаваемая формулой

$$
\mathscr{H}(f(t ; u))=\left[\bigcup_{h \in \mathbb{R}}\{f(t+h ; u)\}\right]_{\Xi_{1}},
$$

компактна в $\Xi_{1}$. Здесь через $[\cdot]_{\Xi_{1}}$ обозначается замыкание в топологии $\Xi_{1}$. Аналогично определяется трансляционная компактность функции $g(t ; x)$ в $\Xi_{2}$.

ОПРЕДЕЛЕНИЕ 3. Символом уравнения (1) называется пара функций

$$
(f(t ; u) ; g(t ; x)):=\sigma(t) .
$$


Предполагается, что $\sigma(t)$ является трансляционно-компактной функцией в $\Xi$. Напомним, что тогда множество $\Sigma:=\left[\bigcup_{h \in \mathbb{R}}\{f(t+h ; u) ; g(t+h ; x)\}\right]_{\Xi}$ компактно в $\Xi$. Последнее равносильно тому, что для любых фиксированных $r>0$ и $M>0$ семейство $\{f(t+h ; u), \partial f(t+h ; u) / \partial t \mid u \in[-r ; r], t \in[-M ; M]\}$ удовлетворяет условиям теоремы Арцела, и семейство функций $\{g(t+h ; x)\}$ равномерно по $h \in \mathbb{R}$ ограничено в $L_{2}(\Omega \times[-M ; M])$, а значит, предкомпактно в слабой топологии.

Имеет место следующее очевидное предложение.

ПреДлОЖЕНИЕ 1. Для любой пары функиий $(\widetilde{f}(t ; u) ; \widetilde{g}(t ; x))$, принадлежсащей $\Sigma$, выполняются условия (3)-(6).

Рассмотрим теперь семейство уравнений

$$
\varepsilon^{2} u_{t t}+\gamma u_{t}+\widetilde{f}(t ; u)=\Delta u+\widetilde{g}(t / \varepsilon ; x),\left.\quad u\right|_{\partial \Omega}=0, \quad x \in \Omega .
$$

Предполагается, что символ уравнения $(9) \sigma(t)=(\widetilde{f}(t ; u) ; \widetilde{g}(t ; x))$ принадлежит $\Sigma$. Из предложения 1 и теоремы 1 следует, что задача $(9),(7)$ имеет хотя бы одно решение, удовлетворяющее (8). Определим пространство, в котором строится траекторный аттрактор $A^{(\varepsilon)}$.

ОПРЕДЕЛЕНИЕ 4. Введем пространство

$$
\mathscr{F}_{+}=\left\{u \in L_{\infty}\left(\mathbb{R}_{+} ; H_{0}^{1} \cap L_{p}\right) \mid u_{t} \in L_{2}^{b}\left(\mathbb{R}_{+} ; L_{2}\right)\right\},
$$

норма в котором определяется по следующей формуле:

$$
\|u\|^{2}=\|u\|_{L_{\infty}\left(\mathbb{R}_{+} ; H_{0}^{1}(\Omega)\right)}^{2}+\|u\|_{L_{\infty}\left(\mathbb{R}_{+} ; L_{p}(\Omega)\right)}^{p}+\sup _{t \geqslant 0}\left\|u_{t}\right\|_{L_{2}(\Omega \times[t ; t+1])}^{2} .
$$

Введем топологию $\Theta_{+}$в пространстве $\mathscr{F}_{+}$. Последовательность $u_{k} \rightarrow u$ в топологии $\Theta_{+}$, если

1) $u_{k} \rightarrow u *$-слабо в $L_{\infty}^{\text {loc }}\left(\mathbb{R}_{+} ; H_{0}^{1}(\Omega)\right)$ и слабо в $L_{p}^{\text {loc }}\left(\mathbb{R}_{+} ; L_{p}(\Omega)\right)$;

2) $\left(u_{k}\right)_{t} \rightarrow u_{t}$ слабо в $L_{2}^{\text {loc }}\left(\mathbb{R}_{+} ; L_{2}(\Omega)\right)$.

ОПРЕДЕЛЕНиЕ 5. Пусть $K_{\Sigma}^{+}(N)$ - множество всех ограниченных (в смысле неравенства (8)) решений уравнения (9):

$$
K_{\Sigma}^{+}(N)=\left\{u(t ; x) \mid Z(t) \leqslant R_{\kappa}+N e^{-\kappa t}\right\} .
$$

Очевидно, что $K_{\Sigma}^{+}(N)$ ограниченное подмножество $\mathscr{F}_{+}$и, следовательно, замкнутое в топологии $\Theta_{+}$. На $K_{\Sigma}^{+}(N)$ действует трансляционная полугруппа $T(t)$ по следующей формуле:

$$
T(h) u(t ; x)=u(t+h ; x) .
$$

Очевидно, что если $u(t ; x)$ - решение уравнения $(9)$ с символом $\sigma(t) \in \Sigma$, то $u(t+h ; x)$ является решением уравнения (9) с символом $\sigma(t+h)$.

ОПРЕДЕЛЕНИЕ 6. Множество $P \subset K_{\Sigma}^{+}(N)$ назовем притягивающим, если для любой его окрестности $O(P) \subset \Theta_{+}$и для любого ограниченного в $\mathscr{F}+$ множества $B \subset K_{\Sigma}^{+}(N)$ существует $t_{0}>0$ такое, что если $t \geqslant t_{0}$, то $T(t) B \subset O(P)$. 
ОПРЕДЕЛЕНИЕ 7. Траекторным аттрактором уравнения (1) называется множество $A^{(\varepsilon)}$, удовлетворяющее следующим условиям:

1) $A^{(\varepsilon)}$ компактно в $\Theta_{+}$;

2) $A^{(\varepsilon)}-$ притягивающее множество для $K_{\Sigma}^{+}(N)$;

3) множество $A^{(\varepsilon)}$ инвариантно относительно полугруппы сдвигов $T(t)$ :

$$
T(t) A^{(\varepsilon)}=A^{(\varepsilon)} \quad \forall t>0 .
$$

Отметим, что слабая топология $\Theta_{+}$сильнее чем, например, $L_{2}^{b}\left(\mathbb{R}_{+} ; L_{2}(\Omega)\right)$. Поэтому, если имеет место притяжение к $P$ в топологии $\Theta_{+}$, то оно имеет также место в $L_{2}^{b}\left(\mathbb{R}_{+} ; L_{2}(\Omega)\right)$.

Теорема 2. Полугруппа $T(t)$ имеет траекторный аттрактор $A^{(\varepsilon)} \Subset \Theta_{+}$.

ДоКАЗАТЕЛЬСтво этой теоремы аналогично соответствующему доказательству, приведенному в [1].

Аналогичные утверждения имеют место также для уравнения (2). Для него задается начальное условие

$$
\left.\bar{u}\right|_{t=\tau}=u_{0} \in H_{0}^{1} \cap L_{p}(\Omega) .
$$

Для этой задачи предполагается выполнение условий (3)-(5) и, кроме того,

$$
f(t ; u+v)-f(t ; u) \geqslant-C v \quad \forall v \geqslant 0 .
$$

Тогда имеет место следующая теорема.

ТЕОРема 3. Пусть выполнены условия (3)-(5), (11) и $\bar{g}(x) \in L_{2}(\Omega)$. Тогда задача $(2),(10)$ имеет единственное решение $\bar{u}(t ; x) \in L_{\infty}\left(\mathbb{R}_{+} ; H_{0}^{1} \cap L_{p}(\Omega)\right)$, причем $\bar{u}_{t} \in$ $L_{2}^{b}\left(\mathbb{R}_{+} ; L_{2}(\Omega)\right)$, и имеет место неравенство

$$
Z(t) \leqslant C Z(0) e^{-\kappa t}+R_{\kappa},
$$

где $Z(t)$ определено в (8) ик то же число, что и в теореме 1.

ДокАЗАТЕЛЬСтво существования решения $\bar{u}(t ; x)$ задачи $(2),(10)$ проводится методом Галеркина, аналогично тому, как это делается в теореме 1.

Как показано в [2], уравнение (2) имеет глобальный аттрактор; следовательно, оно имеет также траекторный аттрактор $A$, обладающий свойствами, аналогичными свойствам траекторного аттрактора $A^{(\varepsilon)}$.

Перейдем к изучению поведения $A^{(\varepsilon)}$ при $\varepsilon \rightarrow 0$. Предполагается, что существует функция $\psi(z ; x)$, для которой выполнено следующее условие:

$$
g(t / \varepsilon ; x)-\bar{g}(x)=\varepsilon \frac{\partial \psi(t / \varepsilon ; x)}{\partial t}, \quad \psi(z ; x) \in L_{2}^{b}\left(\mathbb{R} ; L_{2}(\Omega)\right) .
$$

ПреДЛОЖЕНИЕ 2. Пусть функиия $g(t / \varepsilon ; x)$ удовлетворяет условию (12). Тогда условие (12) имеет место также для любой функиии $\widetilde{g}(t / \varepsilon ; x)$, принадлежащей $\mathscr{H}(g(t / \varepsilon ; x))$, для любого $0<\varepsilon \leqslant 1$. 
ДокАзАтЕЛЬСтво. Из определения 2 следует, что функцию $\widetilde{g}(x ; t / \varepsilon)$ можно представить в следующем виде:

$$
\widetilde{g}(x ; t / \varepsilon)=\lim _{k \rightarrow \infty} g\left(x ; t / \varepsilon+h_{k}\right),
$$

где $h_{k}$ - некоторая числовая последовательность. Отсюда и из (12) следует

$$
\widetilde{g}(x ; t / \varepsilon)-\bar{g}(x)=\lim _{k \rightarrow \infty}\left(g\left(x ; t / \varepsilon+h_{k}\right)-\bar{g}(x)\right)=\lim _{k \rightarrow \infty}\left(\varepsilon \frac{\partial \psi\left(x ; t / \varepsilon+h_{k}\right)}{\partial t}\right) .
$$

Предел в правой части этого равенства существует, так как $\widetilde{g}(x ; z) \in L_{2}^{b}\left(\mathbb{R} ; L_{2}(\Omega)\right)$. Следовательно, выполнено равенство

$$
\lim _{k \rightarrow \infty}\left(\varepsilon \frac{\partial \psi\left(x ; t / \varepsilon+h_{k}\right)}{\partial t}\right)=\varepsilon \frac{\partial \widetilde{\psi}(x ; t / \varepsilon)}{\partial t} .
$$

Предложение доказано.

Основным результатом этой работы является следующая теорема.

Теорема 4. Пусть выполнено условие (12), а также условия теоремы 3 и теоремы 1. Тогда траекторный аттрактор $A^{(\varepsilon)}$ уравнения (1) стремится $к$ аттрактору А уравнения (2) в топологии $\Theta_{+}$.

В доказательстве теоремы 4 используются идеи, изложенные в книге [1].

\section{СПИСОК ЦИТИРОВАННОЙ ЛИТЕРАТУРЫ}

[1] V. V. Chepyzhov, M. I. Vishik, Attractors for Equations of Mathematical Physics, American Mathematical Society Colloquium publications, 49, American Mathematical Society, Providence, RI, 2002.

[2] А. В. Бабин, М. И. Вишик, Аттракторы эволюиионных уравнений, Наука, М., 1989.

\section{А. С. Ляпин}

Российский государственный технологический университет им. К.Э. Циолковского (МАТИ)

E-mail: andser2001@yandex.ru
Поступило 16.03.2007 